\title{
Computer Simulation and Optimization to Solve Groundwater Flow Problems
}

\author{
Oleg Stelia ${ }^{\mathrm{a}}$, Igor Stelia ${ }^{\mathrm{b}}$
}

${ }^{a}$ Faculty of Computer Science and Cybernetics, Taras Shevchenko

National University of Kyiv, Ukraine

b EPAM Systems, Kyiv, Ukraine

E-mail: oleg.stelya@gmail.com, igor.stelia@gmail.com

Ukraine 2021 


\section{Hydrological computer system KRISFLOW}

KRISFLOW HCS is designed for determination of the depth of groundwater depending on various factors, in particular agricultural practices and drainage system regime. The system is associated with the geographic information system which allows to systematize data about the long-term regime of flooding and predict the dynamics of groundwater in accordance with natural, agricultural, and technological factors and to justify solutions on optimization of drainage networks.

Adaptation the model to hydrogeological conditions of areas with large planar dimensions needs coordination and introduction of a large number of input data of different nature, which is presented in the form of tables and maps. For irrigation systems, for example, KRISFLOW requires data for areas and rules of irrigation, location of channels of different purposes and water losses for filtration, location of boreholes, data on the pumping volumes from them, monitoring data, etc. Data input is greatly simplified with the user interface of application and geographic information system (GIS). 
Informational System has sophisticated user interface for data input with the help of screen forms with visual control in GIS. In addition, binding of the models and GIS with its ability to process data and spatial analysis functions significantly enhances data analysis, display of simulation results, creation of thematic maps, etc.

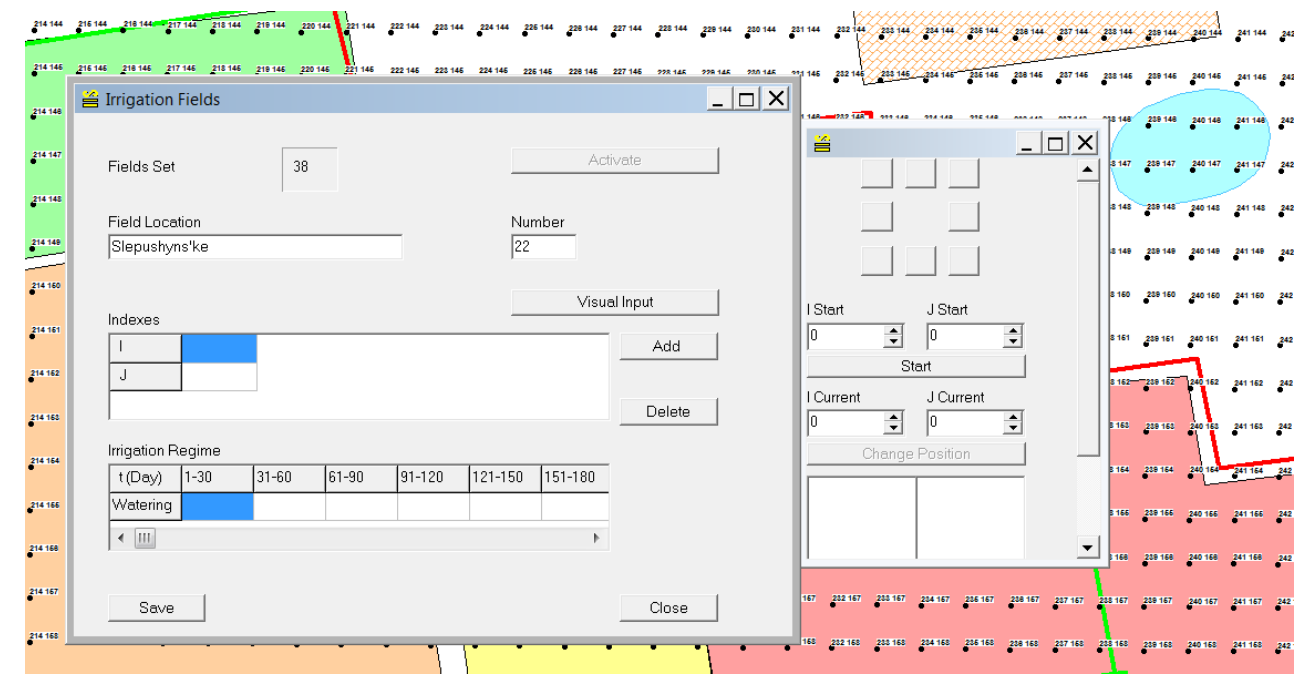

The developed software allows automated dis-playing of simu-lation results on a map in the form of absolute ground water surface levels or thematic maps according to the depth of occurrence of the groundwater, as well as graphic and attribute information of the model. In addition, the information system has convenient means of setting the object of investigation, which allows quick adaptation to the conditions of different areas which require actions to prevent flooding and control of influence of the harmful effects of ground water. 
Using the informational system as a whole or its individual components allows:

- Making a long-term forecast of flooding, using the data of area of irrigation, average rainfall, irrigation rate and location of drainage wells.

- Optimizing the network of observation wells.

- Working out various schemes of reconstruction of irrigation and drainage system on the model.

- Implementing the model calculation of the situation development with groundwater in the region.

- Reducing the negative impact associated with the infiltration losses of irrigation water.

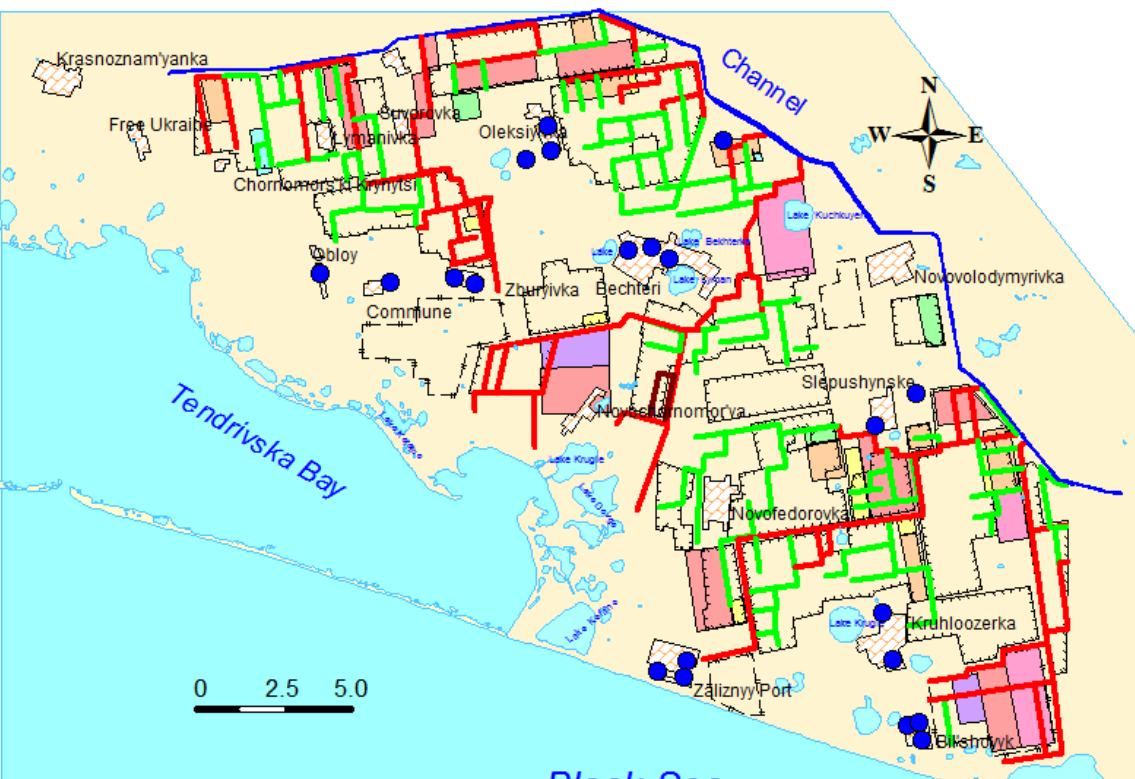

Black Sea 
The informational system was used to simulate the dynamics of groundwater and optimize drainage at Krasnoznamenskaya irrigation system, located in the Golopristanskiy district, Kherson region. The simulation of flooding up to 2020 was made.

The developed methodology and informational system comply with globally accepted approaches for solving of problems associated with harmful effects of water.

Using the elements of the system, the influence of tailing dumps on the groundwater and the flow of pollution into the Dnieper from the Poltava Mining and Processing Plant river was modeled (in conjunction with the SNCLavalin Group Inc. within the framework of Assessment of Groundwater Pollution with Industrial and Municipal Wastes project).

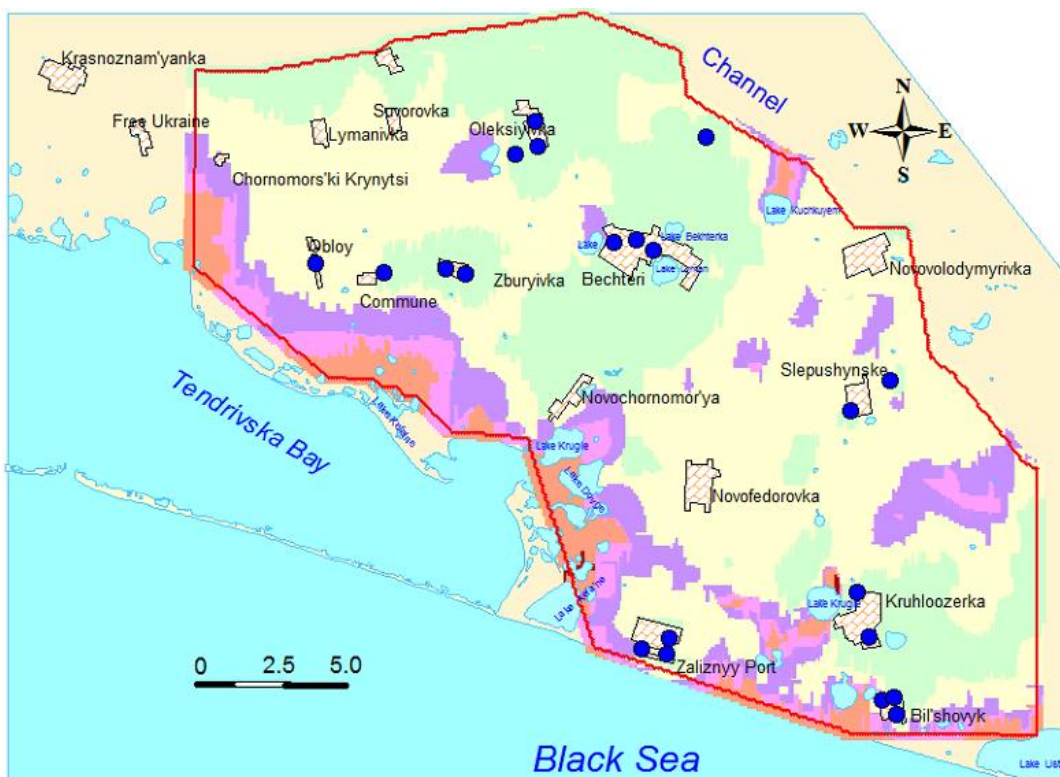

Example of thematic map for calculated groundwater occurrence. 
Evaluation of Contaminants Transport from Storage and Burial Places of Industrial to the Dnieper River by Groundwater

The project goal was improving of surface and groundwater quality, which were contaminated due to technogeneous leakage from different waste disposal sites (tailings storage, accumulating ponds etc.) in the lower part of the Dnieper river basin. As a result of the project measures aimed for reduction of filtration losses of contaminants from wastes storage to underground watersand to the Dnieper river were developed for the tailings storage of the Poltava Mining and Processing Plant.

They allowed to decrease a risk of environmental contamination and to increase ecological resistance of drinking water supply by increasing a volume of underground water consumption and decreasing usage of contaminated

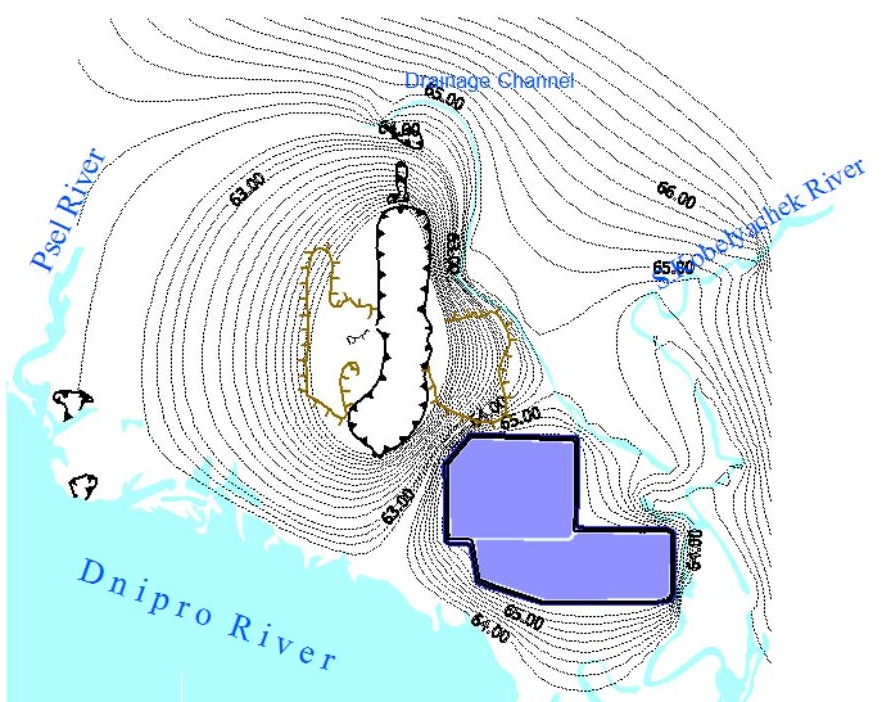
surface waters. 
The results of the project are:

- database with hydrogeological, engineering, geological, geochemical and another data, necessary for creation of a filtration and migration model, as well as for long-term monitoring of the object;

- a filtration and migration model for forecasting contaminants transport from disposal sites of liquid industrial and house-holding wastes with groundwater and their transfer to surface water;

- decision support for selection of engineer constructions for underground and surface water protection on the example of the Poltava Mining and Processing Plant;

- maps with ecological and geological content, including: a map of condition of tap-water sources in the zone of influence of the Poltava MPP; a map of sources of contamination of underground and surface waters; a map of technogeneous changes of geological medium; a map of forecast on ecological and hydrogeological conditions changes in accordance with modeling results;

- substantiation and selection of an optimal scheme of engineer measures aimed at protection ground- and surface waters from contamination. 


\section{Drainage Optimization Model}

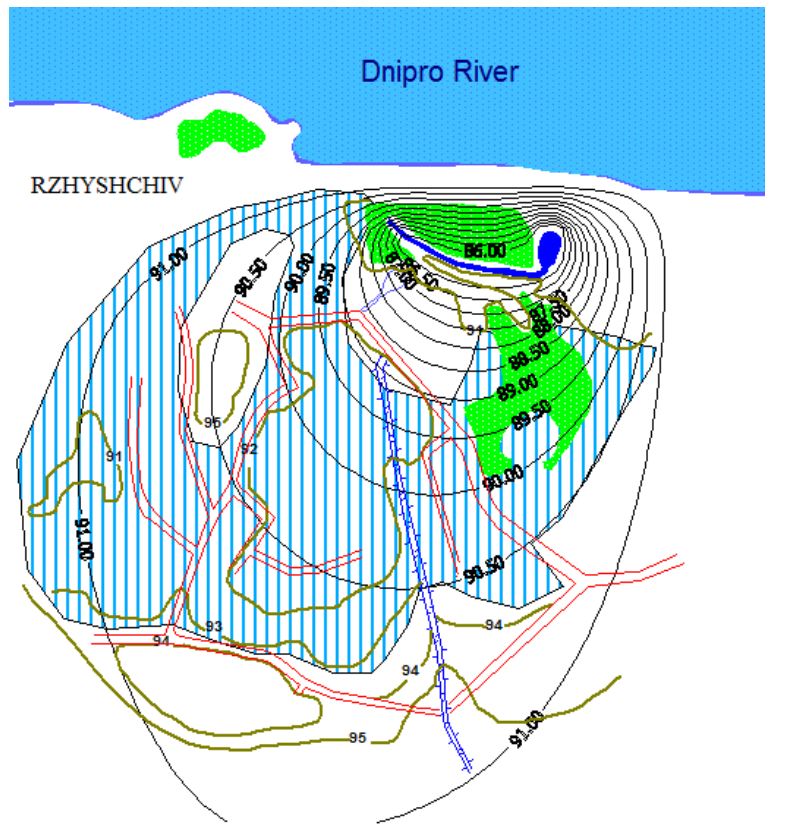

The model is designed for improvement of drainage schemes and development of optimal drainage regimes for areas flooded by groundwater.

This model allows:

- determining the optimal drainage system regime (water pumping rate providing the normal groundwater level) depending on data in monitoring wells;

- carrying out the decisionmaking support upon drainage design.

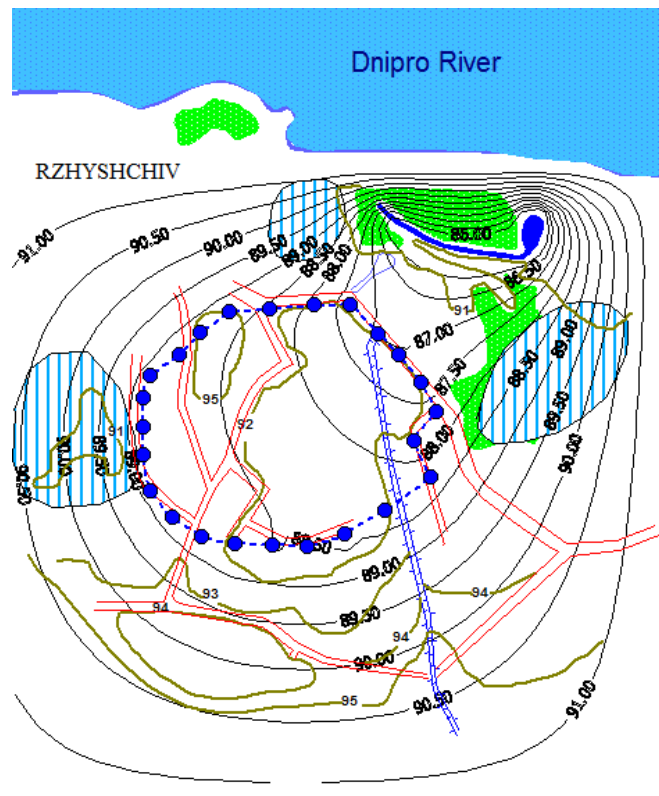




\section{Identification of Groundwater Pollution Sources}

The problem of identifying source function for equation of pollution transport is regarded. The transition to the problem of optimal control for stationary and non-stationary equations with partial derivatives is carried out. The adjoint problems are constructed. For minimizing correspondent functional, the gradient method is applied. The difference method is used for solving the direct and the adjoint problem on the each step of the gradient method.

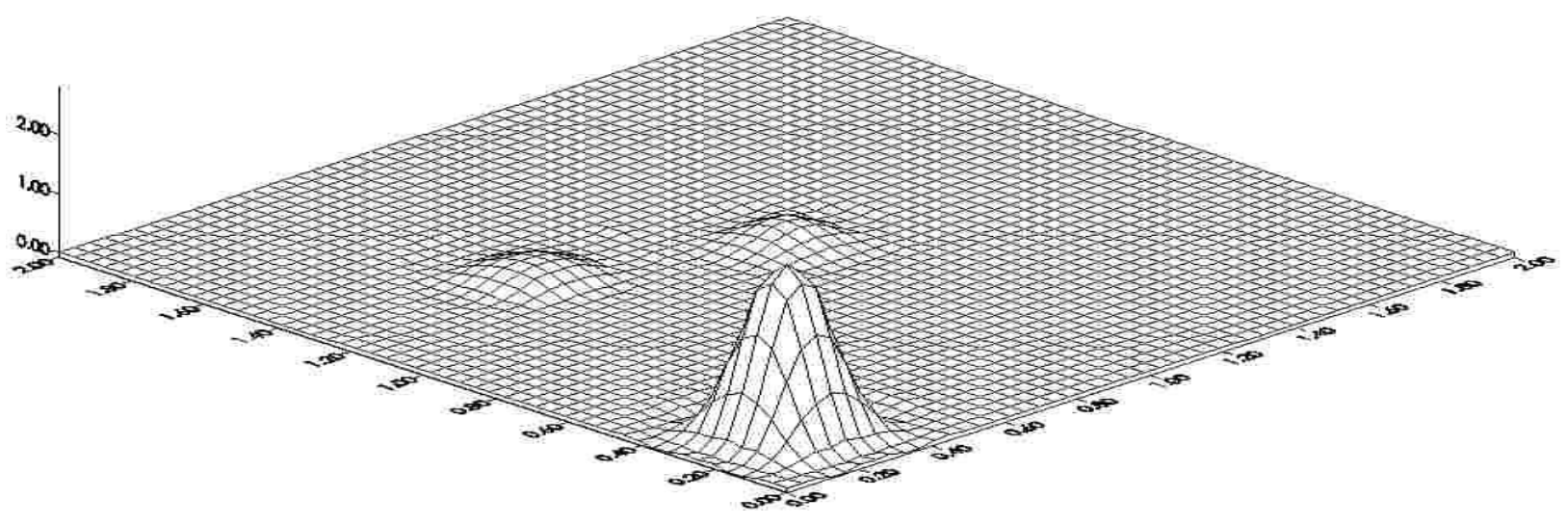

